\title{
7 Using MEESTAR for early evaluation of ethical, legal and social implications of a socio-technical support system for mechanically ventilated patients
}

\section{Lessons learned from the ACTIVATE project}

\author{
Angelika Schley, Katrin Balzer
}

\section{Abstract}

In intensive care units (ICU), mechanically ventilated patients undergoing weaning from the respirator represent a highly vulnerable population. To support their early re-orientation and participation, the ACTIVATE project aims to develop and pilot a socio-technical system that facilitates the communication between these patients and the ICU health care team. Such digital health technologies (DHT) need to be assessed in terms of ethical, legal and social implications (ELSI) before they can be introduced in health care practice. In the ACTIVATE project we chose the Model for Ethical Evaluation of Socio-Technical Arrangements (MEESTAR) as guiding theoretical framework to assess relevant ELSI. Based on our intermediate findings and experiences, the objective of this article is to reflect on the applicability of MEESTAR to the assessment of ELSI of support systems targeting the acute care for critically ill patients. Following the Socratic approach, various data sources and research methods are iteratively applied for the ELSI assessment of the ACTIVATE system under development. Numerous positive implications and potential challenges, varying with the perspectives of patients and health professionals, especially nurses, were identified. Based on the preliminary findings and experiences, we expect that the implementation of the Socratic approach in combination with MEESTAR will ensure that relevant ELSI of the ACTIVATE system will be early detected and taken into account in the development and adaptation of this support system.

\subsection{Introduction}

A rising number of digital health technologies (DHT) with various fields of application and diverse user groups are already available or under development (BMG 2019). DHT can be classified by function, ranging from (i) system service technologies such as electronic health records, through (ii) DHT which are intended to help users understand healthy living and illnesses by providing information and resources, to (iii) DHT which aim to prevent and manage diseases, or (iv) DHT that directly aid diagnostics or treatment of diseases, such as implants, robots for surgery or electronic decision aids (NICE 2019). The overall aim of these different technologies is to improve the proce- 
dures and outcomes of health care services (Bräutigam et al. 2017; Daum 2017). However, despite these desired benefits, the echo to this technological transformation of health care is ambivalent due to a number of ethical, legal or social concerns (Kunze 2017). Especially in the area of care for elderly or seriously ill people, DHT may affect sensitive issues related to being human, for instance autonomy or privacy (Assasi et al. 2014; Linke 2015). Furthermore, concerns related to data security or excessive demands on patients or staff members are expressed (Bräutigam et al. 2017).

Technologies are neither neutral nor value-free. Technologies, as they are developed and used, are the result of interests and values of developers and users. These interests and values as well as that of any other individuals involved and the society as a whole have to be taken into account in the development, assessment and implementation of DHT (Wright 2011). Addressing moral issues can increase the transparency of the assessment of the technologies' consequences and allow better informed decision-making about their implementation and reimbursement in health care by early and comprehensive identification and discussion of potential ethical pitfalls (Assasi et al. 2016; Bellemare et al 2018). It is thus of importance to integrate a systematic evaluation of ethical, legal and social implications (ELSI) in early stages of technology development (Brey 2017; Enam et al. 2018; NICE 2019).

The increasing awareness of the need to incorporate an evaluation of ELSI, or more specifically, ethical implications, into the assessment of (digital) health technologies goes along with a growing array of methods used or recommended for conducting such evaluations. Methods reported in the literature differ significantly in terms of philosophical approach, structure and scope (Assasi et al. 2014). Assasi et al. (2014) identified 43 conceptual frameworks or practical guidelines, varying in approach, structure and comprehensiveness. The choice of the framework and methods for data collection and analysis has to take into account the context, the purpose of the analysis and the availability of resources and required organizational capacities.

Emerging technologies are new, innovative and still in development (Brey 2017). Thus, they are still a promise. Evaluation of ethical implications at early technology development stages cannot be conducted on the basis of experiences with an already implemented and therefore entrenched technology. There are no data about existing products, its uses and its impact on ELSI. However, there is also no one right method to evaluate ethical implications of emerging healthcare technologies (Assasi et al. 2016; Hofmann 2008). One potentially suitable model is the Model for the Ethical Evaluation of Socio-Technical Arrangements (MEESTAR). MEESTAR was developed during a ten-month study in 2012. The main aims of the study were to identify key ethical problems taken into consideration with regard to the use of systems for ambient assistance and care in elderly people and, furthermore, to provide researchers, developers, suppliers and users of those systems with a tool that enables them to identify ethical challenges and discuss them constructively (Manzeschke et al. 2015). 


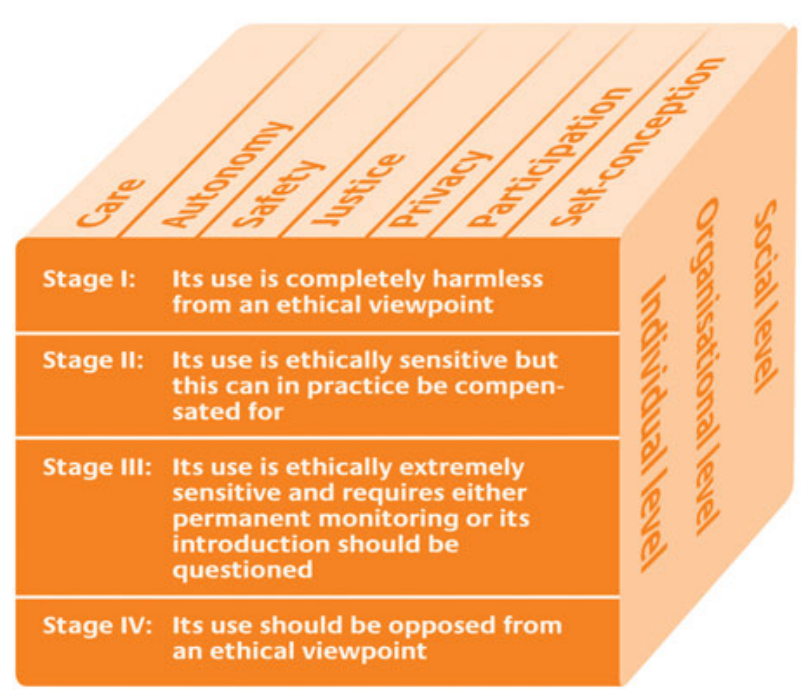

Fig. 7.1: MEERSTAR: $x$-axis: dimension of ethical evaluation; $y$-axis: stages of ethical evaluation; z-axis: levels of ethical evaluation (Manzeschke et al. 2015).

The MEESTAR model comprises three axes as shown in figure 7.1. On the x-axis seven ethical dimensions are presented which were identified as being essential for the assessment of ambient social support systems: care, autonomy, safety, justice, privacy, participation and self-conception. The y-axis represents an assessment scheme to classify the overall ethical threats related to the technology of interest. It consists of four ordinal-ranked categories, with the lowest (best) being "its [the system's] use is completely harmless from an ethical viewpoint" and the highest (worst) being "its [the system's] use should be opposed from an ethical viewpoint". The z-axis reflects the focus of assessment: individual people, organizations, or the society.

For the application of MEESTAR to a technology under evaluation, Manzeschke et al. (2015) offer detailed explanations and a set of questions related to each ethical dimension. In the final analysis two key questions shall be answered: is the use of the given system ethically doubtful or harmless? And, can the identified ethical problems be mitigated or even resolved before completion of technology development? To make sure that ethical challenges resulting from technological functions of the socio-technical system of interest can be eradicated, MEESTAR should always be used in an iterative manner throughout the development and evaluation process (Manzeschke et al. 2015).

Although MEESTAR was primarily developed for social-technical systems targeting the home care setting, it may also help guide the evaluation of ethical and other legal or socio-cultural implications of digital technologies developed for use in other health care settings such as acute hospital care. Due to a lack of alternative models, we chose MEESTAR as theoretical framework for the evaluation of ELSI in the ACTIVATE project. The acronym ACTIVATE stands for a multi-disciplinary project aiming to develop and pilot an Ambient System for Communication, Information 
and Control in Intensive Care (https://projekt-activate.de). The project specifically addresses the communication needs of patients who undergo weaning from invasive mechanical ventilation during the treatment in intensive care units (ICU). Patients receiving mechanical ventilation are not able to express themselves verbally due to an endotracheal tube or tracheal cannula. Both the patients and the health care staff, especially nurses, perceive the communication with each other as challenging and often frustrating (Bergbom-Engberg and Haljamăe 1993; Guttormson et al. 2011; Holm et al. 2018), particularly since effective devices to facilitate this communication are lacking (Happ et al. 2011). However, ineffective communication is distressing to many ventilated critically ill patients, especially during the highly vulnerable process of being weaned off from the ventilator (Rose et al. 2014; Tsay et al. 2013), and assumed to increase the risk of deterioration and complications such as delirium (Nilsen et al. 2014).

Therefore, the core objective of the ACTIVATE system is to support the early communication between ventilated ICU patients while undergoing weaning from the ventilator and nurses who usually have the closest and most frequent contact to them during this process and thus allow more sufficient patient-nurse communication and patient involvement in nursing and medical care. The basic assumption is that this facilitation of communication will improve patient-relevant outcomes such as re-orientation and patient participation or the risk of adverse events, and reduce nurses' work-related burden.

The technical infrastructure of the ACTIVATE system is being designed based on the results of initial user and context analyses, user preference studies and joint workshops of project members (Kordts et al. 2018). In its current shape it consists of an ensemble of newly developed devices, among them a Ball-shaped Interaction and Rehabilitation Device (BIRDY) for data input by ventilated patients in very early weaning stages, a monitor screen as output device as well as speakers and headphones for auditive output. In clinical practice, the ACTIVATE system will be applicable via three major routes: first, the patients can directly activate the support system by themselves and use it to express their symptoms, wishes and other needs while undergoing weaning from the respirator. Second, nurses, other health professionals and relatives can use specific applications of the ACTIVATE system at the bedside to get into contact easier with the patient, gather more patient-reported information about her or his symptoms and needs, and thus develop a better understanding of her or his experience during the mechanical ventilation and weaning procedures. Third, the support system allows to provide the patient regularly with auditive and visual information, e. g. about the actual date, place of treatment or primary nurse, and with music or other preferred stimuli to help early re-orientation. These three routes can be used independently from each other but may also be combined, depending on the patients' cognitive and communication abilities. Fig. 7.2 illustrates how they are assumed to be implemented in clinical practice. 




Fig. 7.2: Possible interaction between a patient and ACTIVATE $^{20}$ (Kordts et al. 2018).

The ACTIVATE project is being conducted by a multi-disciplinary team involving university partners from information, nursing and health sciences, the nursing research and IT service units and several ICUs of a university hospital, as well as industry partners for the design, manufacturing and later distribution of the support system. The three-year project follows the principles of human-centered design (ISO 2009) and includes three main phases: requirement analysis, development and piloting, and clinical evaluation. Throughout all phases, potential ELSI are repeatedly assessed, discussed and taken into account in upcoming development and evaluation steps. A multi-disciplinary advisory board, including experts from nursing science, intensive medicine, psychology, speech therapy, data security and patient representatives, is involved in the evaluation of ELSI through annual meetings and telephone conferences. Based on the experiences and research findings accumulated in the AC-

20 The patient holds BIRDY in her left hand. Through rotating BIRDY left or right, she or he can navigate through the menu structure and, for example, select the item for pain to communicate current burden due to pain. If the nurse is at the bedside, the patient will be able to communicate via the ACTIVATE screen with the nurse. For situations when the nurse is not in the patient's room, the nurse will be equipped with a smartphone to get information about the patients' interaction with the system and about the type and content of the signals sent by the patients via BIRDY. After receiving a message that the patient has selected the item "pain", the nurse will visit the patient to get into direct contact for communication. Another feature of the ACTIVATE system is that relatives will have the opportunity to upload patients' own music, photos and videos into the system to stimulate patients' re-orientation and support their emotional well-being. 
TIVATE project so far, this paper aims to reflect on the applicability of the MEESTAR model for identification and discussion of ELSI related to the ACTIVATE system, i. e. a socio-technological support system that targets another health care setting and user groups than those ambient support systems originally addressed by the model.

\subsection{Methods}

For the continuing assessment of potential ELSI, a combination of iterative inquiries using different methods and involving various target groups and stakeholders is used in the ACTIVATE project. Methodologically, this stepwise process is based on the Socratic approach as recommended by Hofmann et al. (2014). The conceptual planning of data collection, data analysis as well as discussion of the findings was guided by the MEESTAR model. Fig. 7.3 provides an overview of the chronological order of the conducted and remaining evaluation steps, including the data sources used. A detailed description of the methods used for ELSI assessment within the ACTIVATE project will be published elsewhere. This chapter summarizes key information on the methods applied in the evaluation steps carried out so far.

The identification and evaluation of ethical implications and challenges within the ACTIVATE project started right at the beginning to assure the earliest possible identification especially of perceived risks related to the use of ACTIVATE. As a first step of the ethical evaluation, relevant stakeholders were identified. These include the main target groups of the ACTIVATE system, i. e. mechanically ventilated ICU patients and ICU nurses, as well as physicians, therapists and relatives who were also identified as being prospective users of ACTIVATE. Other relevant stakeholders important for the technology development were also identified, such as computer scientists, data protection experts or speech therapists. All of these stakeholders' perspectives will be considered throughout the evaluation of ELSI, either by respective study samples or by the members of the project team and the advisory board.

As a next step, baseline data regarding the target groups' expectations about the potential benefits, challenges and harms related to the intended ACTIVATE support system and potentially relevant context factors were gathered and analyzed within the first six project months. Various information sources, comprising both secondary (aggregated) and primary data, were used in this evaluation step. For secondary data analysis, two scoping reviews were conducted to get an overview of existing research evidence on (i) the views and experiences of mechanically ventilated patients and informal caregivers during the ICU stay and (ii) digital technologies developed to facilitate the communication with ICU patients who are unable to orally verbalize their needs, symptoms or wishes. Each scoping review consisted of systematic literature searches in several electronic databases and complementary searches via Google Scholar and reference lists of eligible articles, followed by systematic two-step selection of relevant articles and standardized data extraction (Tricco et al. 2018). The 
primary data collection comprised (i) 10 semi-structured, non-participating observations by members of all project team partners in two ICU wards, (ii) semi-structured topic-guided face-to-face interviews with 16 ICU patients directly after being successfully weaned off from a respirator and 16 relatives of such patients, (iii) three focus groups with 26 nurses, one physiotherapist, and one chaplain, and (iv) semi-structured topic-guided face-to-face interviews with 6 ICU physicians (Henkel et al. 2018). The main purpose of the observations was to ensure that key members of all project partners share a basic understanding of the ICU care environment, the goals and procedures of nursing and medical care for ventilated patients and the symptoms and needs of patients undergoing weaning from mechanical ventilation. Both the focus groups and the interviews aimed to provide deeper insights into the perceptions, experiences and expectations of the patients, relatives (informal caregivers) and hospital staff related to the weaning process, the challenges in the patient-nurse, patient-staff or patient-family communication during this process, and potential advantages or disadvantages of digital support of this communication. All interviews were recorded as audio files and then transcribed verbatim. The data gathered from the qualitative inquiries were thematically analyzed and aggregated with the findings from the scoping reviews. An inductive-deductive approach was used for this thematic analysis, with the ethical dimensions of the MEESTAR model serving as theoretical framework for the grouping of inductively identified themes relevant to ethical or related implications.

In a successive step, the preliminary list of potential ELSI arising from this analysis was discussed with the project team and the multi-disciplinary advisory board in a structured workshop using the World Café method to ensure that the perspectives of all participants are sufficiently reflected. This workshop took place approximately 11 months after project start. The questions recommended for ethical reflection by the MEESTAR model and the Socratic approach were used to guide through the discussions. Based on the workshop results, the preliminary ELSI list was revised and fed back to all workshop participants, including those responsible for the development of the technical infrastructure of ACTIVATE.

To keep abreast with the advancing of the technology development, qualitative inquiries were repeated several times in order to validate the preliminarily identified ELSI against the detailed personas, application scenarios and technical features developed in the meantime. Until now, two focus groups involving 10 nurses and two physiotherapists, and one speech therapist were conducted in project month 15 , and another two involving 10 nurses in project month 28. For project month 31, individual interviews with former ICU patients undergoing weaning from the respirator are planned. The participants were/will be asked to discuss, with an ethical viewpoint in mind, potential challenges and benefits of the ACTIVATE system. Again, the interview audio files were /will be transcribed verbatim and then analyzed by means of the same methods as described above. In addition, two further structured discussions 


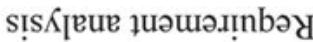

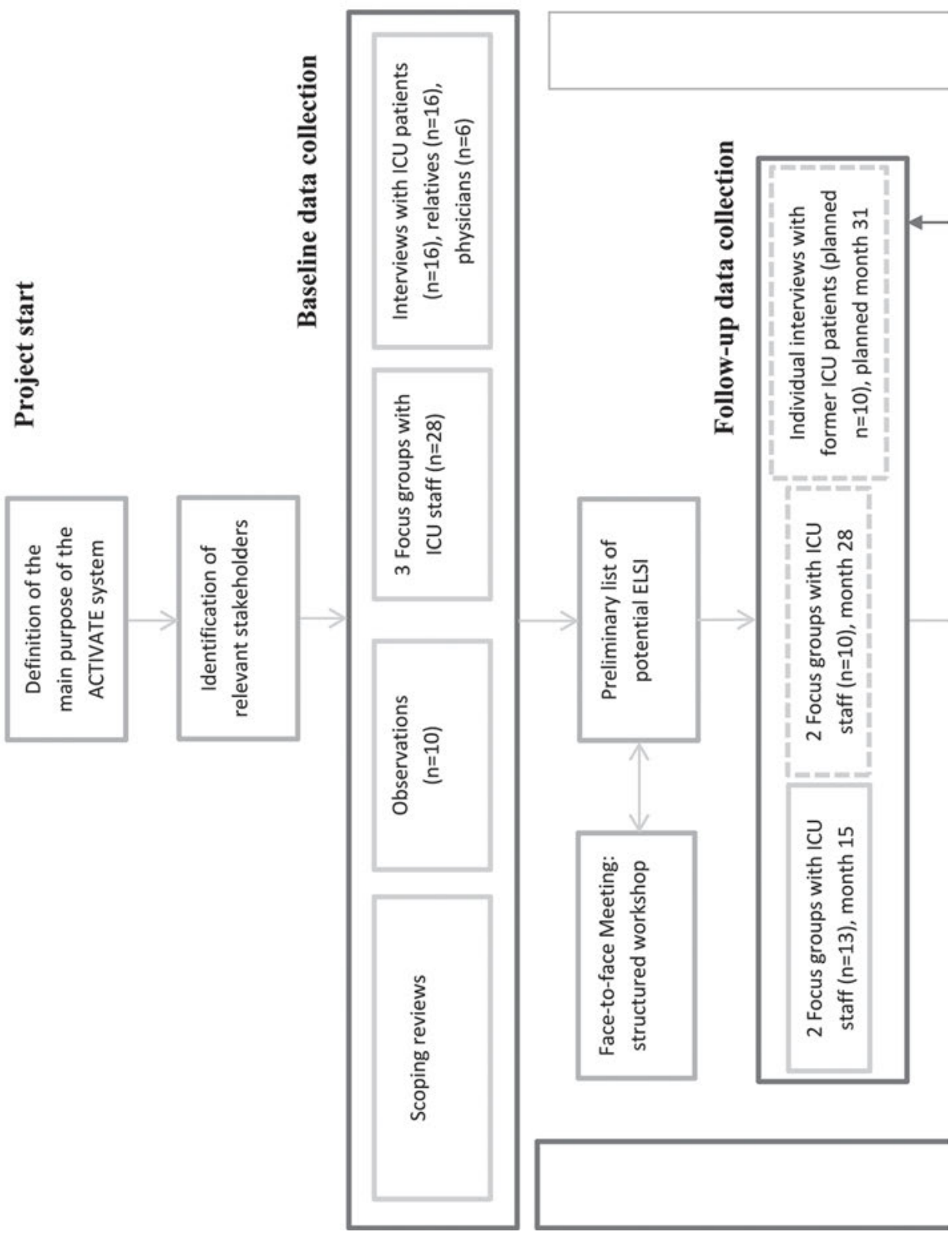

Fig. 7.3: Relevant steps of ethical evaluation within the ACTIVATE project. 


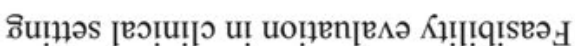

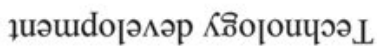

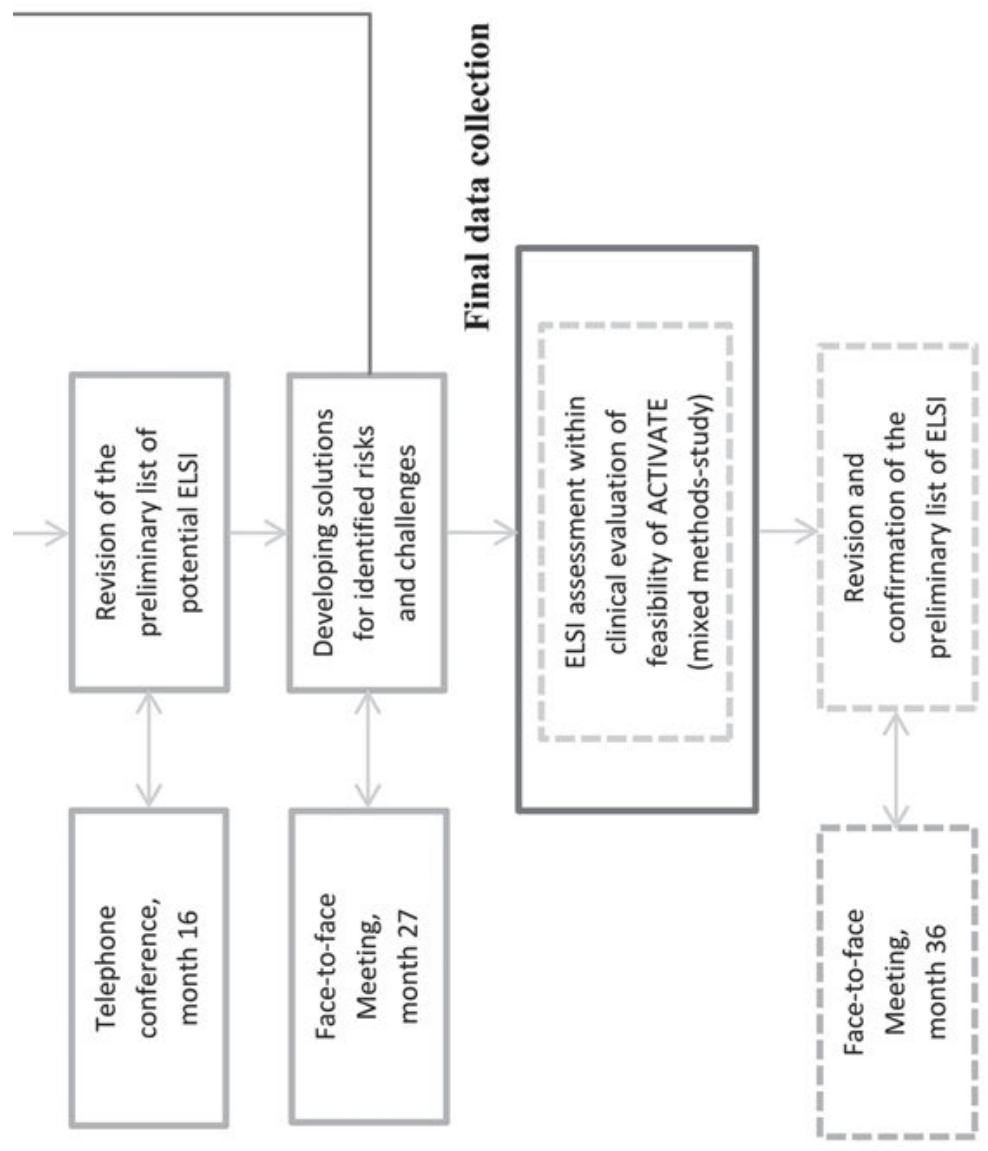


of the preliminary ELSI list with the advisory board, took place, one via telephone conference and one face to face.

In the final step, the iteratively list of potential ELSI will be taken into account during the clinical evaluation of the feasibility of the ACTIVATE system during the project months 33 to 36 . For this study a mixed methods design will be used, consisting of a cohort study complemented with semi-structured non-participating observations of patient-nurse communication episodes, focus groups with nurses and other professionals, semi-structured interviews with patients and a survey among ICU patients' relatives. The protocol for this study is still under development. However, results of this study will also be used for a final discussion of a reflection on the list of ELSI together with the advisory board.

\subsection{Results}

In this chapter, we report a summary of intermediate findings from the analysis and the within-project discussion of the data gathered until project month 15 (figure 7.3). While the findings presented below are still preliminary, they already reflect a synthesis of the views and expectations of all stakeholders involved in the early ELSI evaluation of the ACTIVATE system under development. In the iterative process of data analysis and discussions with the project team and advisory board members, it emerged that the ELSI of the intended support system partially differ between the stakeholders. In particular, two major points of views have to be distinguished: that of the patients targeted by the ACTIVATE support system and that of the health professionals, especially nurses, intended to use this system in their care for these patients. Therefore, themes emerging from the data analyses and discussions were grouped according to these two perspectives. Although the findings assigned to each perspective are rooted in an amalgam of all stakeholders' views and expectations, it has to be recognized that the perspective of the patients is mainly informed by the primary and secondary data directly gathered from (former) ICU patients with weaning experience and their informal caregivers (relatives), while the staff perspective largely reflects the findings from the focus groups and interviews with nurses and other ICU health professionals carried out in this project. Until now, our analyses and discussions did not provide indication of ELSI uniquely linked to informal caregivers. Instead, the views and expectations expressed by these caregivers or by others taking on their perspectives largely resemble those found for the patients' perspective and were therefore assigned to this perspective as well. The staff perspective is dominated by data gathered from nurses who were purposively included in a larger number in the qualitative inquiries than other professionals since they represent the main target group of the ACTVATE system among the ICU staff. However, the ELSI derived from the whole body of data analyzed so far are quite consistent across the various professions involved in our studies and discussions. Therefore, no distinctions were made between the pro- 
fessions in the synthesis of the preliminary findings, and the terms "staff perspective" and "nurses' perspective" will be used synonymously in the following.

The evaluation of ELSI revealed numerous implications, positive and negative, with regard to the prospective use of the ACTIVATE system. Not all ethical dimensions of the MEESTAR model appeared to be of equal importance from each perspective. While from the patients' perspective, all of the seven dimensions were noted except justice, the staff perspective was not linked to three dimensions: care, justice and participation. Thus, based on our preliminary findings, the dimension justice is not viewed as being relevant from either perspective. It was indeed mentioned that the ACTIVATE system shall be available to every ICU patient undergoing weaning, and it was also stressed that all staff members shall have access to this system, but this is more a requirement for the future than an ethical issue at this time of the technical development. The ACTIVATE support system, once implemented, is planned to be part of the routine hospital infrastructure. Thus there shall be no access restrictions due to financial reasons.

The dimension care was, as already mentioned, not raised as a topic relevant to ELSI from the staff perspective. But from the patients' perspective, both positive and negative implications associated to this dimension were brought up. On the one hand, there is the expectation that patients' needs and symptoms will be better recognized and met when the ACTIVATE system is in use. On the other hand, concerns were expressed that this support system might replace nurses as a communication partner, leading to a reduction of patient-nurse communication and hampering the building of trustful relationships between patients and staff.

The dimension privacy was only associated with implications related to data protection, from both perspectives. It was acknowledged that the ACTIVATE system will record and process personal und sensitive health-related data. Particular data protection threats were noted due to the risk that with the ACTIVATE system in use patients may generate data without being aware of it, especially at early weaning stages when they have not yet fully regained their consciousness.

Like the MEESTAR dimension care, participation was only linked to the patients' perspective. Furthermore, it is the only dimension which was exclusively viewed positively. The expectation is expressed that the ACTIVATE system will assist patients to participate in decisions affecting their treatment and care. Furthermore, the support system is expected to enhance the communication between the patients and their informal caregivers, provide access to individual media as music, photos or videos and facilitate remote participation in family life.

The dimension self-conception includes different implications depending on the perspective taken on. From the staff perspective, it is understood as professional self-conception with regard to the field of nursing. The identified implications reflect ambivalent views and expectations, varying between an improved job satisfaction through enhanced patient-nurse communication and accordingly optimized care on the one hand and deep concerns that the ACTIVATE system might replace nurses 
through taking over the communication with the patients on the other hand. Self-conception from the patients' perspective is understood as the feeling of being an active agent despite all of the impairments and restrictions entailed by the critical health conditions and the invasive treatments in ICU care. Being able to communicate, to participate and therefore to take on active roles, instead of being merely a passive recipient of nursing care and medical treatments, is being viewed as a positive implication from the patients' perspective. The feeling of helplessness should be reduced to a minimum. However, the patients' perspective includes some ambivalence, too, since concerns were also expressed that the ACTIVATE system may hurt the individual self-concept of patients not willing to communicate by means of a technological system.

Regarding the dimension autonomy there is one strong positive implication from the patients' perspective: the possibility for the patients to communicate wishes and needs and to choose whether or not to communicate them as well as how to communicate them. Empowerment and self-determined acting and communicating are the desired benefits related to this dimension. But some concerns were also raised, among them the risk that a patient might feel forced to use the ACTIVATE system. Furthermore, patients admitted as an emergency case cannot be made familiar with the support system ex ante, they may be not able to provide their informed consent to the use of this system right at admission. Both the positive and the negative implications noted regarding this dimension are relevant to patients and the ICU staff

Positive and negative implications were mentioned, relating to the dimension safety. Positive implications mainly affect the patients' perspective and are associated with an optimized patient-nurse or patient-staff communication, respectively, which may lead to earlier and more accurate symptom assessment, more appropriate responses to patients' needs and therefore to an optimized nursing care and medical treatment. A major concern from the patients' perspective is that the ACTIVATE system might overstrain the patients due to its various features and related visual or auditory stimuli. The risk of reduced monitoring of the patients was also mentioned. Staff might rely on the patients' ability to use ACTIVATE and eventually reduce their efforts for patient observation and nursing assessment. Further concerns mentioned from the patients' perspective relate to the risk of developing a device-related pressure ulcer or an elevated exposure to radiation. From the staff perspective, similar concerns were noted as from the patients' perspective, altogether resulting into the worry that the ACTIVATE system may put excess demands on the staff, especially nurses, on top of the already existing workload and technical systems in ICU care. 


\subsection{Discussion}

The early evaluation of ELSI related to the prospective use of the ACTIVATE system indicated numerous positive implications but also potential challenges. To summarize, from the patients' perspective clear-cut benefits in terms of almost all MEESTAR dimensions are expected from the ACTIVATE system, with participation being exclusively viewed positively. With regard to potential negative implications, a number of threats were noted from the patients' perspective as well, many of them affecting the dimension's safety, autonomy and privacy. From the staff perspective, the preliminary list of ELSI includes potential benefits and risks regarding the MEESTAR dimensions self-conception, privacy, autonomy and safety. For some of these implications, the underlying considerations resemble those behind the patients' perspective as they are also linked to the assumed impacts of the ACTIVATE system on the patient-nurse communication. From both perspectives, the potential of communication improvements by implementation of this support system has been recognized and judged as being relevant in terms of ELSI, for example by facilitating a more person-centered care and thus enhancing the participation, safety and autonomy of the patients as well as nurses' work satisfaction and professional self-conception. On the other hand, potential downsides related to the effects on the patient-nurse communication were also mentioned and discussed. A major concern is that the socio-technical support system may replace face-to-face communication between patients and nurses or other health professionals, respectively, by patient-machine communication, thus leading to a reduction of direct patient-nurse contacts at the bedside. From the patients' perspective this may increase the risk of insufficient symptom and needs assessment and thus comprise the safety, participation and autonomy of the patients. Furthermore, from both perspectives the idea of patient-machine communication partially substituting patient-nurse communication challenges fundamentals of nursing care and, from the nurses' perspective, nurses' professional self-conception. Also, across both perspectives concerns were brought up that implementation of the ACTIVATE support system may change the normative standards of preferred routes of communication, with socio-technical support systems becoming compulsory to use in the care for highly vulnerable patients such as ICU patients undergoing weaning, irrespective of individual preferences for or against this mode of communication.

All of the ELSI noted so far for the ACTIVATE system could be classified under one of the ethical dimensions of MEESTAR. No implications were detected which refer to ethical, legal or socio-cultural subjects not covered by this model. Thus, our preliminary findings suggest that MEESTAR provides a suitable framework to guide the early evaluation and adaptation of the ACTIVATE system in terms of ELSI although it was originally not conceptualized for the assessment of socio-technical support systems to use in acute care settings (Manzeschke et al. 2015). This indicates that the MEESTAR model is less context-bound than expected. Instead, our findings underscore that it is based on ethical considerations and moral values universally required for the use 
of DHT in conditions fit for human beings. However, as all of our inquiries and discussions were mainly guided by MEESTAR, there is still a certain risk that we missed implications of the ACTIVATE system outside this theoretical frame. In our project, we strive to limit this risk by use of the Socratic approach which implies that divergent stakeholders and various data sources are systematically incorporated throughout all steps of ELSI assessment in this project (Hofmann et al. 2014). Furthermore, the Socratic approach includes more than 30 moral questions which we take into account in addition to the ethical dimensions of the MEESTAR model in the collection, analysis and discussion of the assessment data. Therefore, while the focus of our ELSI assessment of the ACTIVATE system is theoretically guided by MEESTAR, our methods aim to ensure that it is not limited to this model.

One ethical and also legally relevant dimension which has to be further examined is privacy. By use of the ACTIVATE system, the promotion of patients' ability to communicate their needs and wishes is exchanged for a loss of privacy related to the generation of data. In MEESTAR, privacy is defined as an inviolable zone established around people (Manzeschke et al. 2015). Following this definition, we have to get more insights into the meaning of privacy from the perspective of ICU patients undergoing weaning from a mechanical respirator and then proceed to discuss how privacy, as understood by the target patients, can be maintained by the ACTIVATE system as much as possible. Another dimension to be discussed further is self-conception from the patients' perspective. Although our findings indicate that ACTIVATE might have positive impacts on patients' self-conception through providing better possibilities to actively participate in care and treatment decisions, the opposing argument was voiced that this support system might have a negative impact on patients' self-conception if patients do not want to use such technology for communication. In the remaining inquiries for the ELSI assessment, we will collect quantitative and qualitative data on patients' responses to the introduction of a prototype of the ACTIVATE system into laboratory and routine ICU care conditions and thus get a more accurate account of patients' concerns and reservations regarding this kind of digital care support.

Aside from the strengths and limitations already discussed above, a further strength of the ELSI assessment within the ACTIVATE project is the continuous involvement of relevant stakeholders from early beginning onwards, among them representatives of the targeted patients and health professionals. Following the framework of participatory technology development, it has to be ensured that technology is developed not only for but also with the target group(s) (Compagna and Derpmann 2009). (Potential) Users might perceive and judge the impact of a technology differently from developers or suppliers. Recent requirements by the National Institute of Health and Care Excellence (NICE) ask developers and suppliers of DHTs to demonstrate that representatives from intended user groups were involved in the design, development and testing of the respective technology. Fulfilment of these requirements is regarded as a minimum evidence standard to increase the acceptability among users (NICE 2019). 
However, in the baseline and early follow-up focus groups and interviews with the (former) patients, informal caregivers and health professionals we noted that it was difficult for them to imagine the actual shape, features and potential applications of the intended ACTIVATE system since at these early project stages we had no prototype at hand and could only present theoretical ideas about the intended design, functions and future use of the ACTIVATE support system. Thus, the very early ELSI assessment has been conducted based on speculative information (Brey 2017). It is thus prone to some uncertainty inherent to these theoretical assumptions and the perception of this information by the study participants, and this uncertainty may limit the validity and completeness of the ELSI revealed by our inquiries so far. But, on the other hand, only this very early ELSI assessment offers the possibility to influence the technology development from the very beginning. The further the technology is developed, the better risks, challenges and benefits can be identified, but it is more complicated to change the technology (Brey 2017). In the remaining assessment steps and especially within a pilot study to evaluate the feasibility of the ACTIVATE system in routine ICU care, a variety of quantitative and qualitative data will be collected from the patients, informal caregivers and health professionals to examine the validity and completeness of the preliminary list of ELSI. For example, we will be able to verify whether the concerns revealed reflect actual potential threats originating from the ACTIVATE system or are rather due to prejudices expressed by individual participants.

\subsection{Conclusion}

This article reports the methods and intermediate results of the early ELSI assessment carried out along the development of the ACTIVATE support system which aims to facilitate the early communication between ICU patients undergoing weaning from a mechanical respirator and the health professionals, especially nurses. In particular, this article aimed to reflect on the question whether the MEESTAR model chosen to theoretically guide this ELSI assessment is suitable for the assessment of a socio-technical support system targeting the acute care for critically ill patients.

So far, several positive implications as well as perceived risks of the ACTIVATE system have been identified by our ELSI assessment. Although not all dimensions of the MEESTAR model seem to be of equal importance for the use of the ACTIVATE system, both from the patients' and the staff perspectives, all of the identified implications could be categorized into one of the seven MEESTAR dimensions. This underscores the universal relevance of the ethical dimensions reflected by MEESTAR and suggests that this model is also applicable to DHT targeting the setting of ICU care. As the ELSI assessment has been implemented since the very beginning of the project, all of the identified challenges and risks were and are still being taken into account in the system's development. In the further course of the ACTIVATE project, the preliminary list of ELSI will be subject to further quantitative and qualitative inquiries to ex- 
amine its validity and completeness and thus provide a robust body of knowledge for the final ELSI assessment of the ACTIVATE prototype before this DHT will be released for large scale evaluation and implementation in ICU routine care settings.

\subsection{Ethical Statement}

All studies were conducted in accordance with the ethical standards of the institutional research committee (file number 17-098). Informed consent was obtained from all individual participants included in the study.

\subsection{Acknowledgment and funding information}

We would like to thank the ACTIVATE team members, including Adrienne Henkel, Björn Hussels, Prof. Dr. Nicole Jochems, Jan Kopetz, Börge Kordts, Susanne Krotsetis, Prof. Dr. Andreas Schrader, and Dr. Armin Will for the discussion of ethical, legal and social implications related to the ACTIVATE system. Special thanks to the advisory board members Prof. Dr. Annette Baumgärtner, Dr. Teresa Deffner, Dr. Florian Dismer, Prof. Dr. Maria Deja, Andrea Leinen, Prof. Dr. Elke Muhl, Peter Nydahl, and Felix Schmidt for their helpful comments and debates relating to ELSI. Project ACTIVATE is funded by the German Federal Ministry of Education and Research (Grant no. 16SV7689)

\section{References}

Assasi, Nazila, Jean-Eric Tarride, Daria O’Reilly and Lisa Schwartz. 2016. Steps toward improving ethical evaluation in health technology assessment: a proposed framework. BMC Medical Ethics, 17:34. https://doi.org/10.1186/s12910-016-0118-0.

Assasi, Nazila, Lisa Schwartz, Jean-Eric Tarride, Kaitryn Campbell and Ron Goeree. 2014. Methodological guidance documents for evaluation of ethical considerations in health technology assessment: a systematic review. Expert Rev Pharmacoecon and Outcomes Res., 14(2), 203-220. https://doi.org/10.1586/14737167.2014.894464.

Bellemare, Christian A., Pierre Dagenais, Suzanne K.-Bédard, Jean-Pierre Béland, Louise Bernier, Charles-Étienne Daniel, Hubert Gagnon, Georges-Auguste Legault, Monelle Parent and Johane Patenaude. 2018. Ethics in health technology assessment: a systematic review. Int J Technol Assess Health Care, 34(5):447-457. https://doi.org/10.1017/S0266462318000508.

Bergbom-Engberg, Ingegerd and Hengo Haljamăe. 1993. The communication process with ventilator patients in the ICU as perceived by the nursing staff. Intensive Crit Care Nurs., 9(1): 40-47. https://doi.org/10.1016/0964-3397(93)90008-L.

Bundesministerium für Gesundheit (BMG). 2019. Digitalisierung. Rahmenplan Ressortforschung des Bundesministeriums für Gesundheit. Handlungsfelder und Schwerpunkte 2019-2022. https:// www.bundesgesundheitsministerium.de/fileadmin/Dateien/5_Publikationen/Ministerium/Broschueren/Rahmenplan_Ressortforschung.pdf. Accessed 26 August 2019. 
Bräutigam, Christoph, Peter Enste, Michaela Evans, Josef Hilbert, Sebastian Merkel, and Fikret Öz. 2017. Digitalisierung im Krankenhaus. Mehr Technik - bessere Arbeit? Study Nr. 364, Dezember 2017. Hans Böckler Stiftung. https://www.boeckler.de/pdf/p_study_hbs_364.pdf. Accessed 26 August 2019.

Brey, Philip . 2017. Ethics of emerging technologies. Hansson, Sven Ove (ed.) Methods for the ethics of technology. London: Rowman and Littlefield International, 175-192.

Compagna, Diego and Stefan Derpmann. 2009. Verfahren partizipativer Technikentwicklung (Working Papers kultur- und techniksoziologische Studien). Duisburg: Universität Duisburg-Essen Campus Duisburg, Fak. Für Gesellschaftswissenschaften, Institut für Soziologie. http://nbn-resolving.de/urn:nbn:de:0168-ssoar-216915.

Daum, Mario. 2017. Digitalisierung und Technisierung der Pflege in Deutschland. Aktuelle Trends und ihre Folgewirkungen auf Arbeitsorganisation, Beschäftigung und Qualifizierung. Im Auftrag der DAA-Stiftung Bildung und Beruf. https://www.daa-stiftung.de/fileadmin/user_upload/digitalisierung_und_technisierung_der_pflege_2.pdf. Accessed 26 August 2019.

Enam, Amia, Johanna Torres-Bonilla and Henrik Eriksson. 2018. Evidence-based evaluation of eHealth interventions: systematic literature review. J Med Internet Res; 20(11): e10971. https://doi. org/10.2196/10971.

Guttormson, Jill L., Karin Lindstrom Bremer and Rachel M. Jones. 2015. „Not being able to talk was horrid “: a descriptive, correlational study of communication during mechanical ventilation. Intensive Crit Care Nurs.; 31(3): 179-186. https://doi.org/10.1016/j.iccn.2014.10.007.

Happ, Mary Beth, Kathryn Garrett, Dana DiVirgilio Thomas, Judith Tate, Elisabeth George, Martin Houze, Jill Radtke and Susan Sereika. 2011. Nurse-patient communication interactions in the intensive care unit. Am J Crit Care; 20(2): 28-40. https://doi.org/10.4037/ajcc2011433.

Henkel, Adrienne, Björn Hussels, Jan Kopetz, Susanne Krotsetis, Nicole Jochems and Katrin Balzer. 2018. Nutzer- und Aufgabenanalyse für ein soziotechnisches System zur Unterstützung der Kommunikation und Reorientierung beatmeter Patienten und Patientinnen in Intensivstationen: Ergebnisse und methodische Herausforderungen. In Zukunft der Pflege. Tagungsband der 1. Clusterkonferenz 2018. Innovative Technologien für die Pflege, ed. Boll, Susanne, Andreas Hein, Wilko Heuten and Karin Wolf-Ostermann, 201-206. Oldenburg: BIS.

Hofmann, B, S Droste, W Oortwijn, I Cleenput, and D Sacchini. 2014. Harmonization of ethics in health technology assessment: a revision of the Socratic Approach. Int J Technol Assess Health Care, 30:1, 3-9. https://doi.org/10.1017/S0266462313000688.

Hofmann, BM. 2008. Why ethics should be part of health technology assessment. Int J Tech Assess Health Care, 24:4, 423-429. https://doi.org/10.1017/S0266462308080550.

Holm, Anna and Pia Dreyer. 2018. Nurse-patient communication within the context of non-sedated mechanical ventilation: a hermeneutic-phenomenological study. Nurs Crit Care; 23(2):88-94. https://doi.org/10.1111/nicc.12297.

ISO 9241-210: 2010. Ergonomics of human-system interaction-Part 210: Human-centred design for interactive systems. International Standardization Organization (ISO). Switzerland. 2009.

Kordts, Börge, Jan Patrick Kopetz, Katrin Balzer amd Nicole Jochems. 2018. Requirements for a system supporting patient communication in intensive care in Germany. In Zukunft der Pflege. Tagungsband der 1. Clusterkonferenz 2018. Innovative Technologien für die Pflege, ed. Boll, Susanne, Andreas Hein, Wilko Heuten and Karin Wolf-Ostermann, 131-136. Oldenburg: BIS.

Kunze, Christophe. 2017. Technikgestaltung für die Pflegepraxis: Perspektiven und Herausforderungen. Pflege \& Gesellschaft, 22. Jg. H.2: 130-145. Weinheim: Beltz.

Linke, Anna F. 2015. Autonomie bei technischen Assistenzsystemen. Ein Trade-off zwischen Privatheit, Unabhängigkeit und Sicherheit. In Technisierung des Alltags. Beitrag für ein gutes Leben? ed Weber, Karsten, Debora Frommeld, Arne Manzeschke, Heiner Fangerau, 179-194 , Stuttgart: Steiner. 
Manzeschke A, Weber K, Rother E, Fangerau H. 2015. Results of the study 'Ethical questions in the area of age appropriate assisting systems'. Ludwigsfelde: Thiel.

National Institute for Health and Care Excellence (NICE). 2019. Evidence standards framework for digital health technologies. https://www.nice.org.uk/Media/Default/About/what-we-do/our-programmes/evidence-standards-framework/digital-evidence-standards-framework.pdf. Accessed 26 August 2019.

Nilsen, ML, SM Sereika, LA Hoffman, A Barnato, H Donovan, and MB Happ. 2014. Nurse and patient interaction behaviors' effects on nursing care quality for mechanically ventilated older adults in the ICU. Res Gerontological Nursing; 7(3): 113-25. https://doi. org/10.3928/19404921-20140127-02.

Rose, Louise, Katie N. Dainty, Joanne Jordan, and Bronagh Blackwood. 2014. Weaning from mechanical ventilation: A scoping review of qualitative studies. Am J Crit Care, 23(5), 54-70. https:// doi.org/10.4037/ajcc2014539.

Tricco AC, Lillie E, Zarin W, O'Brien KK, Colquhoun H, Levac D, Moher D, Peters MDJ, Horsley T, Weeks L, Hempel S, Akl EA, Chang C, McGowan J, Stewart L, Hartling L, Aldcroft A, Wilson MG, Garritty C, Lewin S, Godfrey CM, Macdonald MT, Langlois EV, Soares-Weiser K, Moriarty J, Clifford T, Tunçalp Ö, Straus SE. 2018. PRISMA Extension for Scoping Reviews (PRISMA-SCR): Checklist and Explanation. Ann Intern Med., 169(7):467-473. https://doi.org/10.7326/M18-0850.

Tsay, Shwu-Feng, Pei-Fan Mu, Shirling Lin, Kai-Wei Katherine Wang, and Yu-Chih Chen. 2013. The experiences of adult ventilator-dependent patients: A meta-synthesis review. Nursing and Health Sciences, 15(4), 525-533. https://doi.org/10.1111/nhs.12049.

Wright, David. 2011. A framework for the ethical impact assessment of information technology. Ethics and Information Technology, 13(3): 199-226. Doi: 10.1007/s10676-010-9242-6. 